\title{
Improved model predictive load frequency control of interconnected power system with synchronized automatic generation control loops
}

\author{
Abdullahi Bala Kunya ${ }^{1 *}$ (D, Mehmet Argin², Yusuf Jibril ${ }^{1}$ and Yusuf Abubakar Shaaban ${ }^{3}$
}

\begin{abstract}
Background: Automatic generation control (AGC) of multi-area interconnected power system (IPS) is often designed with negligible cross-coupling between the load frequency control (LFC) and automatic voltage regulation (AVR) loops. This is because the AVR loop is considerably faster than that of LFC. However, with the introduction of slow optimal control action on the AVR, positive damping effect can be achieved on the LFC loop thereby improving the frequency control. In this paper, LFC synchronized with AVR in three-area IPS is proposed. Model predictive controller (MPC) configured in a dense distributed pattern, due to its online set-point tacking is used as the supplementary controller. The dynamics of the IPS subjected to multi-area step and random load disturbances are studied. The efficacy of the developed scheme is ascertained by simulating the disturbed system in MATLAB/Simulink.
\end{abstract}

Results: Based on the comparative analysis on the system responses, it is established that by cross-coupling the LFC loop with AVR, reductions of $66.45 \%$ and $59.09 \%$ in the frequency and tie-line power maximum deviations respectively are observed, while the respective settling times are found to be reduced by $29.68 \%$ and $22.77 \%$ when compared with the uncoordinated control scheme. In addition, the standard deviation and variance of the integral time absolute error of the system's responses have reduced by $23.21 \%$ and $20.83 \%$ respectively compared to those obtained in a similar study.

Conclusions: The reduction in the maximum deviations and settling times in the system states indicates that introducing the voltage control via AVR loop has improved the frequency control significantly. While the lower standard deviation and variance of the integral time absolute error signify improvement in the robustness of the developed algorithm. However, this improvement is at the detriment of the controller size and computational complexity. In the uncoordinated control scheme, the control vector is one-dimensional, while in the coordinated scheme, the control vector is two-dimensional for each CA.

Keywords: Area control error, Automatic voltage regulator, Control area, Load frequency control, Model predictive control

\footnotetext{
* Correspondence: abkunya@abu.edu.ng

'Department of Electrical Engineering, Ahmadu Bello University, Zaria,

Nigeria

Full list of author information is available at the end of the article
}

Springer Open (c) The Author(s). 2020 Open Access This article is licensed under a Creative Commons Attribution 4.0 International License, which permits use, sharing, adaptation, distribution and reproduction in any medium or format, as long as you give appropriate credit to the original author(s) and the source, provide a link to the Creative Commons licence, and indicate if changes were made. The images or other third party material in this article are included in the article's Creative Commons licence, unless indicated otherwise in a credit line to the material. If material is not included in the article's Creative Commons licence and your intended use is not permitted by statutory regulation or exceeds the permitted use, you will need to obtain permission directly from the copyright holder. To view a copy of this licence, visit http://creativecommons.org/licenses/by/4.0/. 


\section{Background}

In a geographically expansive interconnected power system (IPS) that comprises of several subsystems better known as control areas (CAs), generation-demand mismatch in any CA can cause frequency deviation in the others CAs. In addition, inter-CA active power flow also deviates from its rated value following this mismatch. As a result, the synchronous speed of the interconnected system of generators is altered which may eventually force them out of synchronism $[1,2]$. To reduce the adverse effect of frequency and inter-CA power flow deviations on IPS operation, a continuous mismatch between power generation and demand must be minimized online. This is achieved by optimal adjustment of generators active power generation (prime mover) through a technique known as load frequency control (LFC), as depicted in Fig. 1. It is an ancillary service in power system operation meant to improve the IPS reliability and quality of its supply [4].

Unlike the frequency that essentially depends on the variation in active power, CA's terminal voltage is majorly affected by change in reactive power. As such, voltage regulation is achieved through optimal regulation of reactive power generation using automatic voltage regulation (AVR). The voltage deviation (from a predefined set point) is measured and augmented into a reactive power command signal. The signal then adjusts the generator field excitation current which eventually regulates the reactive power generation [5, 6], as shown in Fig. 1.

Generally, the time constant of the AVR loop excitation system is quite small compared to that of the prime mover. Thus, LFC loop is slower than the AVR loop due to the mechanical inertia constants in the former. As such, these two control loops are designed to have negligible cross-coupling and treated independently [3, 7]. However, with introduction of slow optimal control action on the AVR, positive damping effect can be achieved on the LFC loop thereby improving the frequency control. In this study, an attempt is made to simultaneously execute the two control loops by slowing the AVR and fast-tracking the LFC loop.

There are numerous studies conducted on either LFC or AVR by applying various control methods like optimal, sub-optimal, robust and intelligent control approaches $[8$, 9]. For instance, in [10], a robust LFC scheme using artificial neural network-based for multi-area system is proposed. In separate but similar studies, proportionalintegral-derivative (PID) controller applied for LFC application is proposed in [11, 12], while a hybridized fuzzy logic-PID controller is developed in [13] for frequency control of IPS. In the study, comparative analysis is carried out between Ziegler-Nichols tuned PID that tuned using heuristic particle swarm optimization (PSO) technique. A similar research work is carried out in [14] by optimizing the fuzzy logic scheduling PI controller parameters with aid of PSO for LFC application. A non-centralized adaptive LFC scheme with aid of mixed $\mathrm{H}_{2} / \mathrm{H}_{\infty}$ is proposed in [9]. An optimal output feedback linear quadratic regulator is proposed in [15]. In various research works like [16, 17], model predictive controller (MPC) has been applied for LFC application in a single area and IPS. A hierarchical double-level MPC based LFC is presented in [18]. The lower level comprises of disperse local PI controllers while the higher control level is made up of supervisory MPC controller which computes set-points for the PIs in the lower control layer to establish coordinated control action.

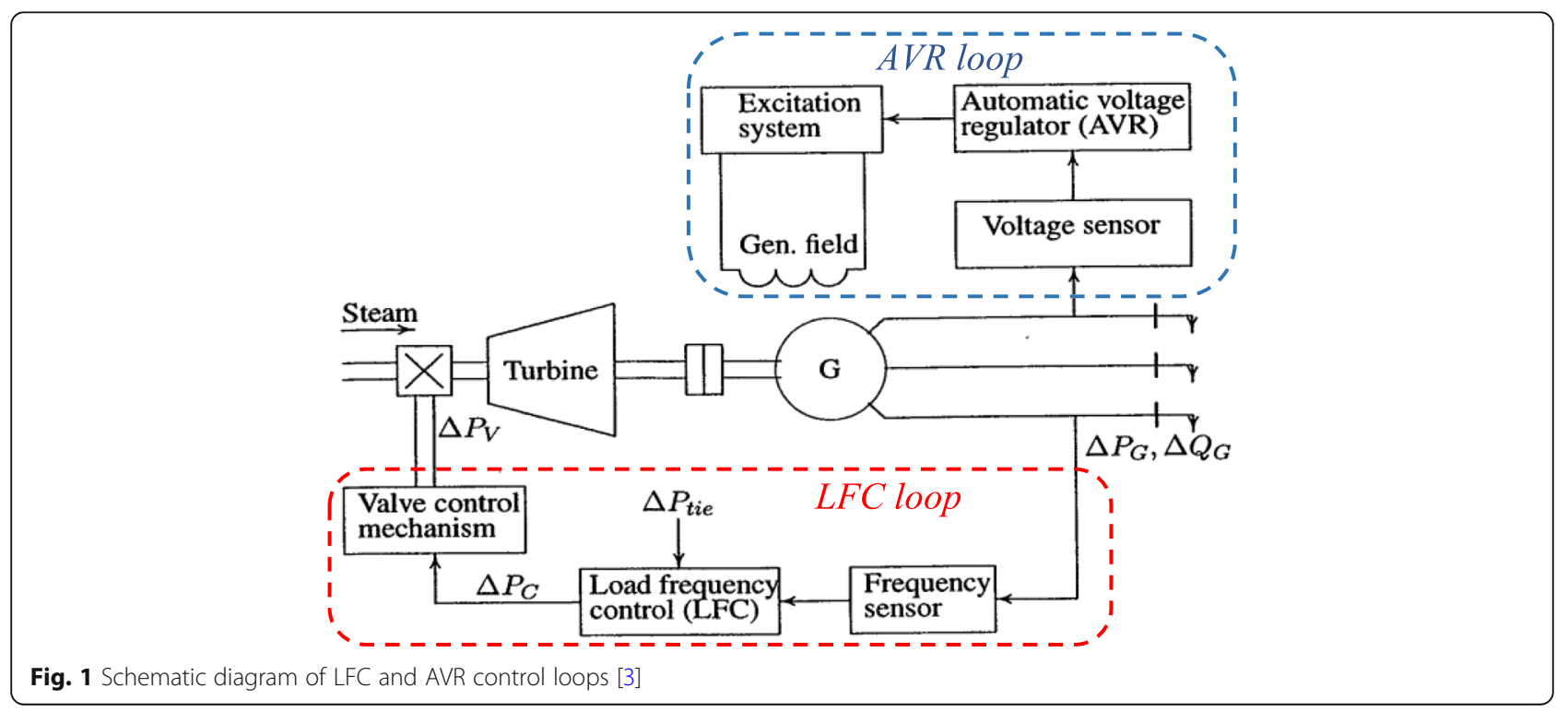


Though PI controller is easy to design and implement, however, suffers from poor handling of system dynamic constraints.

In addition, the effectiveness of these controllers often deteriorates considerably with increase in system complexity and size. This is why many research works focused on improving their robustness by optimizing the gains using heuristic and meta-heuristic optimization techniques. For instance, [19] employed a bacterial foraging optimization algorithm (BFOA) to determine the optimal gains of PI controller and establish its upper hand over genetic algorithm-tuned in a double-CA nonreheat thermal IPS [20]. proposed an LFC of a multiarea thermal power system using grey wolf optimization algorithm (GWO) based classical controllers and changed the cost function to integral of time multiplied by absolute value of error (ITAE). The responses are compared with BFOA and GA optimized ITAE-based PI controller to establish its superior efficacy. Similarly in [21], quasi-oppositional whale optimization algorithm (QOWOA) is employed to tune a dual-mode PI controller serving as supplementary frequency controller. The quasi-oppositional-based learning theory is incorporated with the WOA to make it faster, efficient, and more reliable. The control scheme is applied on a two- and threearea interconnected system with governor dead band nonlinearity. Similar approach is presented in [22]; however, a quasi-oppositional differential search algorithm is applied instead of WOA.

On the other hand, the design of AVR based on $\mu$ controller to enhance IPS stability and enhance power transfer capacity is proposed in [23]. Damping torque approach is employed for tuning the AVR parameters. A novel hierarchical AVR scheme in modern power grids is presented in [24]. It is based on the state-ofthe-art wide-area measurement system technologies to achieve coordinated voltage regulation. In [25], nonlinear regulation for synchronous generator excitation control to carry out AVR is presented. The scheme comprises of the nonlinear interconnection and damping assignment technique to establish the generator voltage feedback AVR.

In spite of the contributions recorded in these literatures (and many more), there are some drawbacks associated with them. Due to the nonlinearities in power system dynamics, the majority of these techniques suffer poor handling of system nonlinearities and violation of system dynamic constraints. In addition, based on the extensive literature review conducted, no attempt is made to study the positive damping impact of cross-coupling AVR with LFC on frequency control of IPS with conventional generators. Though, in some research works like [7, 26, 27], simultaneous voltage and frequency control using various control techniques is proposed, however, they all focused on AC microgrid with inverter-interfaced distributed generators.

In this study, distributed MPC (dis-MPC) is applied for LFC cross coupled with AVR in a 3-CA IPS. The controller is chosen considering its ability to handle system constraints efficiently, predict system future states, and optimize the control action online. Generation rate constraints (GRC), and frequency deviation limits are considered as constraints. This paper makes the following contributions:

i- Proposes an LFC scheme with cross coupling between the LFC loop and AVR loop by slowing the latter and fast-tracking the former loop.

ii- Proposes a dense dis-MPC scheme for LFC in a multi-area interconnected power system that is devoid of excessive offline parameter tuning.

iii- Uses Kalman filter approach to estimate the states of the CAs. Some research works assumed that all the states of the CAs are observable, which is quite unrealistic.

The paper is presented with the mathematical modeling of the IPS in section 2, systematic design of the disMPC for LFC described in section 3. While section 4 describes the simulation results and discussion, with section 5 concluding the paper.

\section{Multi-area IPS mathematical modeling}

The dynamics of multi-area IPS is known for its exceptional complexity and nonlinearity. Due to continuous change in generation-demand balance, IPS states change so frequently within tight tolerance. Moreover, the frequency measured in each control area shows the deviation in tie-line power and the generation-demand mismatch in the area. Thus, the dynamics of IPS while studying LFC needs to consider the tie-line power deviation.

Normally, the LFC scheme in large-scale IPS is carried out by approximating the overall system with several separated subsystem models and executes the control in either distributed or decentralized manner. Distributed control schemes differ from decentralized control as a result of the consideration given to the external state information transmitted from the neighboring subsystems.

The study of LFC is restrained to comparatively low oscillations; the complex nonlinear model of the IPS is derived and linearized [5].

For $i$ th CA in a multi-area IPS shown in Fig. 2, the mismatch between generation, $P^{\mathrm{G}}$ and demand, $P^{\mathrm{D}}$ on frequency, $f_{i}$ is modeled using linearized swing equation as 


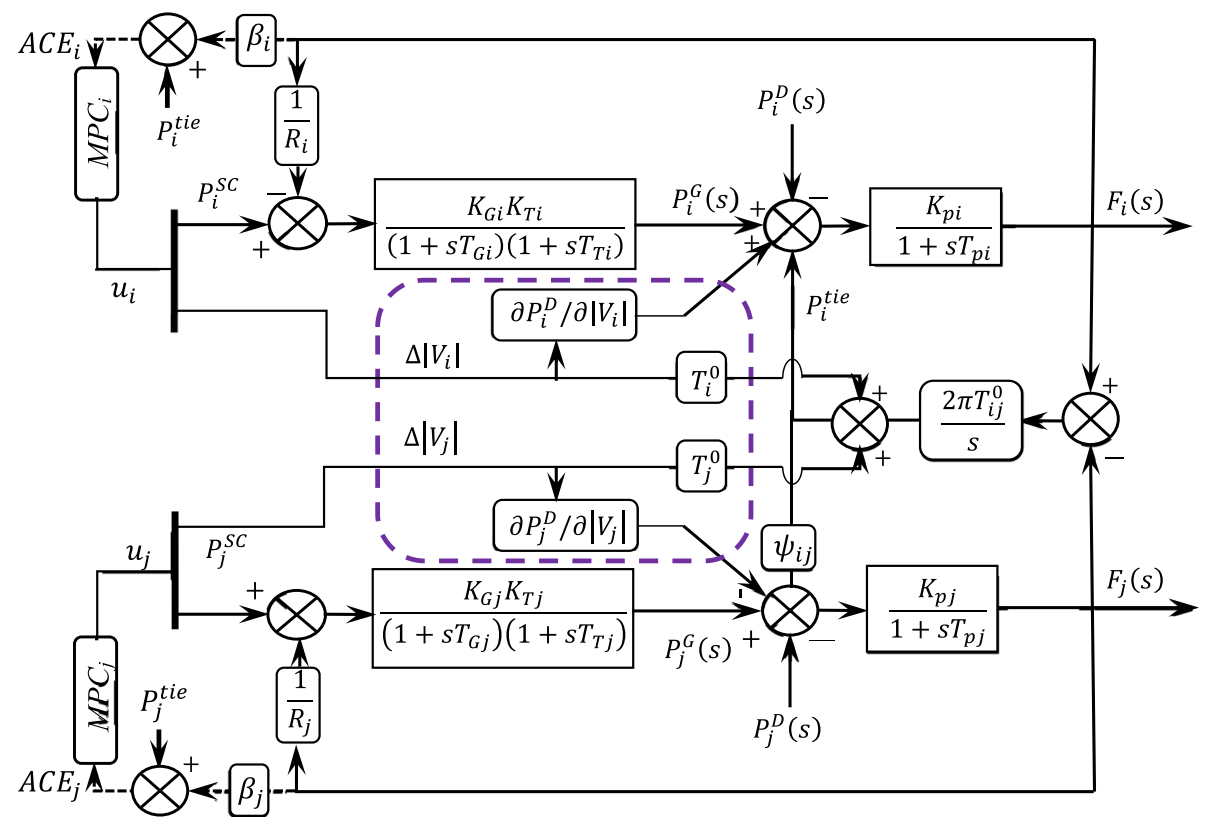

Fig. 2 Modified dynamic model of arbitrary 2-CA IPS

$$
\dot{f}_{i}=\frac{1}{H_{i}}\left(P_{i}^{G}-D_{i} f_{i}-P_{i}^{t i e}-P_{i}^{D}\right)
$$

where $P_{i}^{G}$ is the $i$ th area total generation computed by summing up the power output of all units in the area as illustrated in (2);

$$
P_{i}^{G}=\sum_{m \in G_{i}} P_{i, m}^{G}
$$

where $D_{i}$ and $H_{i}$ are the $i$ th control area damping coefficient and inertia constant respectively. $G_{i}$ is the index set of generators in the $i$ th control area assumed to form a coherent group. This assumption agrees with assigning a single frequency to each control area.

In conventional LFC design, only phase angles, $\delta_{i}$, are changing while the voltage magnitudes, $\left|V_{i}\right|$, are maintained constant. This is due to the fact that AVR loop (responsible for voltage control) is much faster than LFC loop. As such, change in tie-line power is normally modeled as in (3) [6].

$$
\dot{P}_{i}^{t i e}=\sum_{j \in \mathcal{A}_{i}^{t i e}} \dot{P}_{i j} ; \quad \dot{P}_{i j}=2 \pi T_{i j}^{0}\left(f_{i}-f_{j}\right)
$$

$$
P_{i}^{t i e}=\frac{\left|V_{i}\right|\left|V_{j}\right|}{X_{i j}} \sin \left(\delta_{i}-\delta_{j}\right)
$$

Where $T_{i j}^{0}$ and $X_{i j}$ are the synchronizing coefficient and reactance of tie-line between area $i$ and $j . \mathcal{A}_{i}^{\text {tie }}$ denotes the index set of all the control areas connected to the $i$ th area.

However, with the proposed introduction of slow control action, the AVR loop time can be made to be (at least theoretically) as large as that of the LFC loop. As such, a more accurate model accounting for change in frequency (phase angle) as well as change in voltage $\left(\Delta\left|V_{i}\right|\right)$ need to be developed. From (3), the change in $i$ th tie-line power flow is obtained as

$$
\dot{P}_{i}^{t i e}=\frac{\partial P_{i}^{t i e}}{\partial\left|V_{i}\right|} \Delta\left|V_{i}\right|+\frac{\partial P_{i}^{t i e}}{\partial\left|V_{j}\right|} \Delta\left|V_{j}\right|+\frac{\partial P_{i}^{t i e}}{\partial\left|\left(\delta_{i}-\delta_{j}\right)\right|} \Delta\left(\delta_{i}-\delta_{j}\right)
$$

Taking the partial derivative of (5), it becomes

$$
\dot{P}_{i}^{t i e}=T_{i}^{0} \Delta\left|V_{i}\right|+T_{j}^{0} \Delta\left|V_{j}\right|+2 \pi T_{i j}^{0}\left(f_{i}-f_{j}\right)
$$




$$
\begin{aligned}
T_{i}^{0} & =\frac{\left|V_{j}\right|}{X_{i j}} \sin \left(\delta_{i}-\delta_{j}\right) \text { and } T_{j}^{0} \\
& =\frac{\left|V_{i}\right|}{X_{i j}} \sin \left(\delta_{i}-\delta_{j}\right)
\end{aligned}
$$

The frequency deviation expressed as the function of angular displacement of the rotors referenced to the stator;

$$
f_{i}=\frac{\Delta \delta_{i}}{2 \pi}
$$

It can be observed from (7) that voltage perturbations result in added increments in the power. The perturbations are seen to increase the loads in the $i$ th CAs by a quantity $\left(\partial P_{i}^{D} / \partial\left|V_{i}\right|^{)} \Delta\left|V_{i}\right|\right.$, as such this additional term is added as virtual load disturbance. The terms are added at the same point where the actual load disturbances are added. Therefore, the model of the two-area system presented in [6] is modified to incorporate the effect of voltage perturbations as shown in Fig. 2.

The dynamics of the non-reheat turbines (chosen for this study) is governed by the load changes and control input signal computed by the LFC controller. The turbine dynamics of the $m$ th unit in the $i$ th area is formulated as

$$
\dot{P}_{i, m}^{G}=\operatorname{sat}_{P_{i, m}^{\dot{G}, i}}\left\{\frac{1}{T_{T_{i, m}}}\left(P_{i, m}^{G o v}-P_{i, m}^{G}\right)\right\}
$$

where $T_{T_{i, m}}$ and $s a t_{\dot{P}_{i, m}^{G}}{ }^{i}$ are the steam chest time constant and saturation nonlinearity to model GRC of $m$ th generator in the $i$ th control area.

The change in valve position of $m$ th generator as a relation to frequency is approximated to have one time constant, $T_{G o v_{i m}}$ as

$$
\dot{P}_{i, m}^{G o v}=\frac{1}{T_{G o v i, m}}\left(P_{i, m}^{S C}-P_{i, m}^{G o v}-\frac{1}{R_{i, m}} f_{i}\right)
$$

In IPS, deviations in frequency and net tie-line power are the two fundamental variables in the LFC. Their combination referred as area control error (ACE) is utilized as a performance measure and serves as the feedback input signal to the LFC. For $i$ th CA, the ACE is computed as [28];

$$
A C E_{i}=\beta_{i} f_{i}+\Delta P_{i}^{t i e}
$$

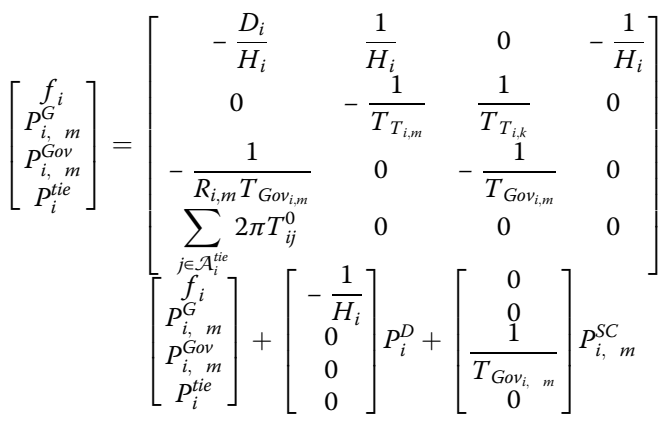

$$
\begin{aligned}
& +\left[\begin{array}{c}
-\frac{1}{H_{i}} \\
0 \\
-2 \pi \sum_{j \in \mathcal{A}_{i}^{t e}}^{0} T_{i j}^{0}
\end{array}\right] f_{j}+\left[\begin{array}{c}
0 \\
0 \\
0 \\
T_{i}^{0}
\end{array}\right] \Delta\left|V_{i}\right|+\left[\begin{array}{c}
0 \\
0 \\
0 \\
T_{j}^{0}
\end{array}\right] \Delta\left|V_{j}\right|
\end{aligned}
$$

$$
\mathrm{ACE}_{i}=\left(\begin{array}{llll}
\beta_{i} & 0 & 0 & 1
\end{array}\right)\left(\begin{array}{llll}
f_{i} & P_{i, m}^{G} & P_{i, m}^{G o v} & P_{i}^{t i e}
\end{array}\right)^{T}
$$

The system model described in (1)-(11) for $i$ th CA is summarized in the state-space model as in (12) and (13). Where $\left[\begin{array}{llll}f_{i} & P_{i, m}^{G} & P_{i, m}^{G o v} & P_{i}^{t i e}\end{array}\right]^{T} \in \mathbb{R}^{4}$ is the state vector $\left(x_{i}\right), P_{i}^{D}$ is the disturbance input while $\mathrm{ACE}_{i}$ is the output for area $i$. The vector $\left[P_{i, m}^{S C} \Delta\left|V_{i}\right|\right]^{T} \in \mathbb{R}^{2}$ is the control input $\left(u_{i}\right)$ comprising of the optimal supplementary control signal, $P_{i, m}^{S C}$ from the LFC loop and the voltage control input, $\Delta\left|V_{i}\right|$ for $i$ th CA. In the conventional LFC scheme, the control vector is one-dimensional $\left(P_{i, m}^{S C}\right)$ while in the developed coordinated scheme, the size of the control vector doubles for each CA because of the introduction of the voltage control input.

\section{Methods}

As mentioned earlier, the aim of this study is to synchronize LFC with AVR in three-area IPS with aid of model predictive controller (MPC) configured in a dense distributed pattern. Slow optimal control action is placed on the AVR in order to achieve a positive damping effect on the LFC loop and eventually improving the frequency control. In this section, the design of dis-MPC-based LFC of the 3-CA IPS developed in the previous section is presented.

\subsection{Distributed MPC design}

The control scheme chosen; MPC is a technique in which the optimal control solution is obtained by solving an online constrained optimization problem, over finite prediction horizon. It is based on an objective function (OF) formulated to penalize the deviations in the system output from the desired trajectory and minimizes the 
control effort. MCP optimization problem is carried out in a discrete pattern, which leads to an array of optimal control inputs. The first entry of the control inputs is applied as the current control action on the system actuators (valves in this context). This approach is repeated in the subsequent time samples until the desired control specifications are met.

MPC consists of two main parts: prediction and control part. The prediction side of the controller is responsible for forecasting the future state of the plant using its present states while the control part uses the predicted states in the OF minimization.

In this work, the MPC is designed with both feed forward and feedback control capabilities. The former is designed to compensate for the measured disturbance prior to attacking the system, while the former is put in place to regain the system stability after destabilizing the system.

As distributed control scheme, each of the three CAs of the system is equipped with an MPC controller, which uses the ACE (and its reference, $A C E_{i}^{\text {ref }}$ taken as zero) as input and based on a given control law, solves for the optimal control solutions as illustrated in Fig. 3.

The continues-time system model described in (12) is first discretized. At time sample $\tau$, an OF consisting of weighted summation of squared tracking errors, control inputs, and the change in control inputs as expressed in (14) is minimized.

$$
\min _{u(0), u(1), \cdots, u\left(N_{c}-1\right)} J_{i}
$$

Such that

$$
\begin{aligned}
J_{i}= & \sum_{k=1}^{N_{p}}(y(\tau)-r(\tau))^{T} Q(y(\tau)-r(\tau)) \\
& +\sum_{k=1}^{N_{p}}(\Delta u(\tau-1))^{T} R_{\Delta u} \Delta u(\tau-1) \\
& +u(\tau-1)^{T} R_{u} u(\tau-1)
\end{aligned}
$$

The first term of (14) represents the cost of output errors while the second and third terms are the cost of input and incremental input respectively.

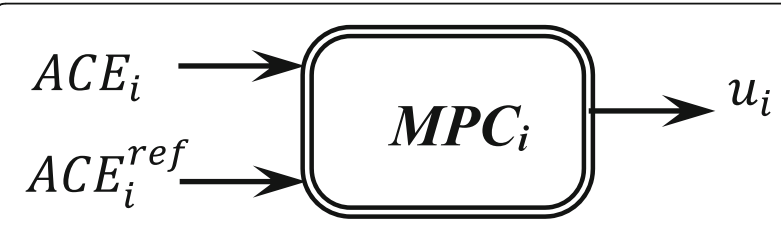

Fig. 3 Schematics of dis-MPC in ith CA

\subsection{System constraints}

Generally, the constraints imposed on the system are the system manipulated inputs, outputs, or state variables. In this study, the objective function in (14) minimized subject to the following constraints:

\section{i- State constraint:}

$$
\begin{aligned}
& x_{i}(\tau+1)=A_{i i} x_{i}(\tau)+B_{i i} u_{i}(\tau)+D_{i i} d_{i}(\tau) \\
& +\sum_{j \in \mathcal{A}_{i}^{\text {tie }}}\left(A_{i j} x_{j}(\tau)+B_{i j} u_{j}(\tau)\right) \\
& y_{i}(\tau)=C_{i i} x_{i}(\tau)
\end{aligned}
$$

ii- Equality constraint: total generated and imported/ exported power must balance out the load demand as expressed in (17).

$$
P_{i}^{G}(\tau)+P_{i}^{t i e}(\tau)=P_{i}^{D}(\tau)
$$

iii- GRC: the generation rate $m$ th unit in $i$ th area is limited to $G R C_{i, m}$ as formulated in (18);

$$
\left|P_{i, m}^{G}(\tau)-P_{i, k}^{G}(\tau-1)\right| \leq G R C_{i, m} ;
$$

A GRC of $0.1 \mathrm{pu} / \mathrm{min}$ is chosen for all the generators.

iv- Frequency fluctuation constraint: The frequency deviations of the individual areas are constrained within certain the minimum and maximum limits of $0.2 \mathrm{~Hz}$ and $-0.2 \mathrm{~Hz}$ respectively as in (19) [17];

$$
\left|f_{i}(\tau)\right| \leq f_{i}^{\xi} ; \quad \text { such that } f_{i}^{\xi}=0.2 \mathrm{~Hz}
$$

Where $N_{c}$ is the MPC control horizon, while $Q, R_{u}$, and $R_{\Delta u}$ in the OF are positive definite and symmetric weighting matrices used to weigh the tracking errors, control inputs, and the change in control inputs. They are tuning parameters selected to realize the preferred performance and can be chosen freely. However, to achieve improved closed-loop performance, weighting matrices optimized using bat-inspired algorithm presented in [29] is used. 
After solving (14) subject to (15)-(19), optimal control solution, $u_{i}$ comprising of $P_{i}^{s c}$ and $\Delta\left|V_{i}\right|$ is obtained and applied to the system as indicated in Fig. 2. Unlike in conventional LFC with $u_{i}$ as one-dimensional, in the proposed study, $u_{i}$ is two-dimensional. As a consequence, the computational complexity of the MPC doubles.

\section{Results}

To demonstrate the effectiveness of the proposed control scheme, a 3-CA, 6-machine (2 thermal generators per CA) IPS, whose parameters are obtained from [18], is designed and controlled with the proposed dense disMPC. Each of the CAs is equipped with a local MPC, which generates the optimal control signal of that particular CA. A sampling interval of $0.1 \mathrm{~s}, N_{c}$ of 20 samples, and a $N_{p}$ of 100 samples are selected as appropriate length to achieve good control performance. The IPS is developed in MATLAB/Simulink and subjected to multi-area step and random load disturbance. The responses of the system are then studied with and without coordinating the two control loops. Settling time and peak deviation are used as performance measures for analyzing the step responses. While integral time absolute error (ITAE) of the ACEs defined in (20) is used to analyze the system responses with random load. ITAE is chosen because it weighs the errors much better after a long time than at the beginning of the response.

$$
I T A E=\int_{0}^{t}\left|A C E_{i}\right| t d t
$$

\subsection{Multi-area step perturbation}

In this scenario, $\mathrm{CA}_{1}$ is subjected to 0.1 puMW step load perturbation at the beginning of the simulation time. $\mathrm{CA}_{3}$ is subsequently subjected to the same load change after $15 \mathrm{~s}$, which $\mathrm{CA}_{2}$ is left unperturbed.

Figure 4 shows the system responses following the MASP. The load disturbance rejection performance of the dis-MPC scheme is evaluated with and without the coordination between the AVR and LFC control loops. It is observed that, in either case, the proposed control scheme has restored the system states to normalcy with approximately zero steady-state error.

However, as it can be observed from Fig. 4, coordinating the two control loops has not only shorten the settling time but also reduced the peak over/undershoot for both load perturbations. Like in $\mathrm{CA}_{1}$ for instance, the proposed coordinated scheme has restored the frequency

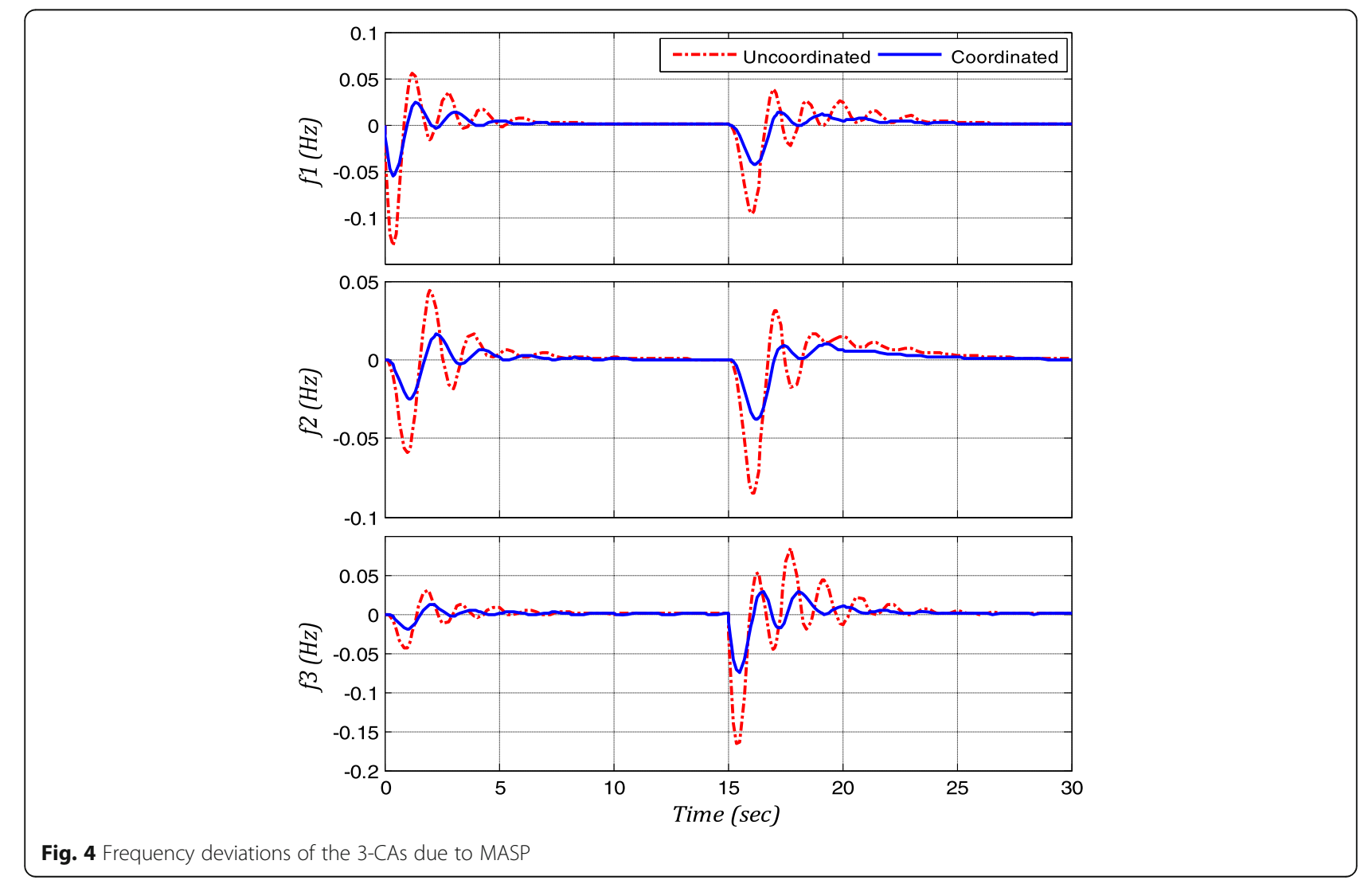




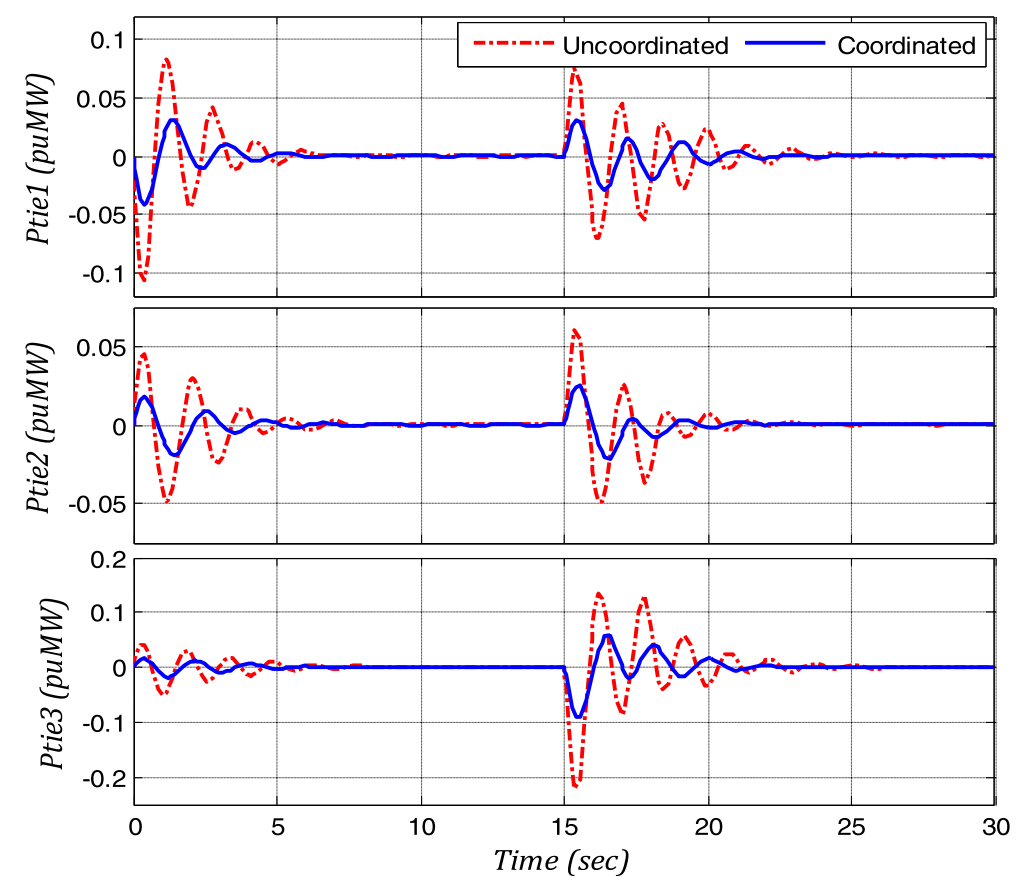

Fig. 5 Tie-line power deviations of the 3-CAs due to MASP

Table 1 Summary of system state responses

\begin{tabular}{|c|c|c|c|c|}
\hline Metrics & States & $A C E_{1}$ (puMW) & $f_{1}(\mathrm{~Hz})$ & $P_{1}^{\text {tie }}$ (puMW) \\
\hline \multirow[t]{3}{*}{ Peak deviation } & Conventional & -0.080 & -0.152 & -0.044 \\
\hline & $\operatorname{dis}-M P C$ & -0.041 & -0.051 & -0.018 \\
\hline & Improvement (\%) & 48.75 & 66.45 & 59.09 \\
\hline \multirow[t]{4}{*}{ Settling time (s) } & Conventional & 7.65 & 7.58 & 6.98 \\
\hline & $\operatorname{dis}-M P C$ & 6.02 & 5.33 & 5.39 \\
\hline & Improvement (\%) & 21.31 & 29.68 & 22.77 \\
\hline & States & $A C E_{2}$ (puMW) & $f_{2}(\mathrm{~Hz})$ & $P_{2}^{\text {tie }}$ (puMW) \\
\hline \multirow[t]{3}{*}{ Peak deviation } & Conventional & 0.019 & -0.058 & 0.041 \\
\hline & dis-MPC based & 0.009 & -0.039 & 0.017 \\
\hline & Improvement (\%) & 52.63 & 32.75 & 58.53 \\
\hline \multirow[t]{4}{*}{ Settling time (s) } & Conventional & 8.46 & 7.86 & 7.43 \\
\hline & dis-MPC & 6.27 & 6.42 & 5.74 \\
\hline & Improvement (\%) & 25.88 & 18.32 & 22.75 \\
\hline & States & $A C E_{3}(\mathrm{puMW})$ & $f_{3}(\mathrm{~Hz})$ & $P_{3}^{\text {tie }}$ (puMW) \\
\hline \multirow[t]{3}{*}{ Peak deviation } & Conventional & 0.023 & -0.047 & 0.046 \\
\hline & dis-MPC & 0.012 & -0.026 & 0.015 \\
\hline & Improvement (\%) & 47.83 & 44.68 & 67.39 \\
\hline \multirow[t]{3}{*}{ Settling time (s) } & Conventional & 6.31 & 6.82 & 6.32 \\
\hline & $\operatorname{dis}-M P C$ & 5.06 & 5.44 & 5.16 \\
\hline & Improvement (\%) & 19.89 & 20.23 & 18.35 \\
\hline
\end{tabular}




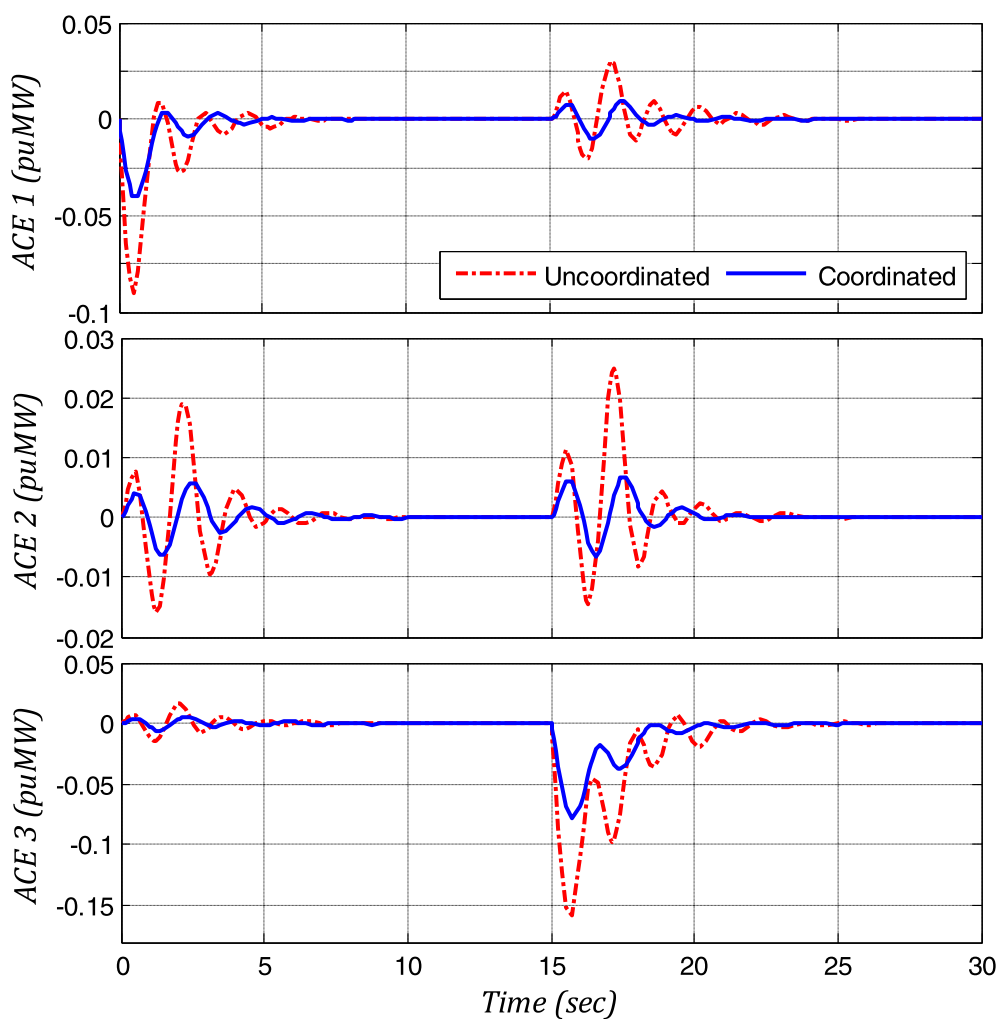

Fig. 6 ACEs of the 3-CAs due to the MASP

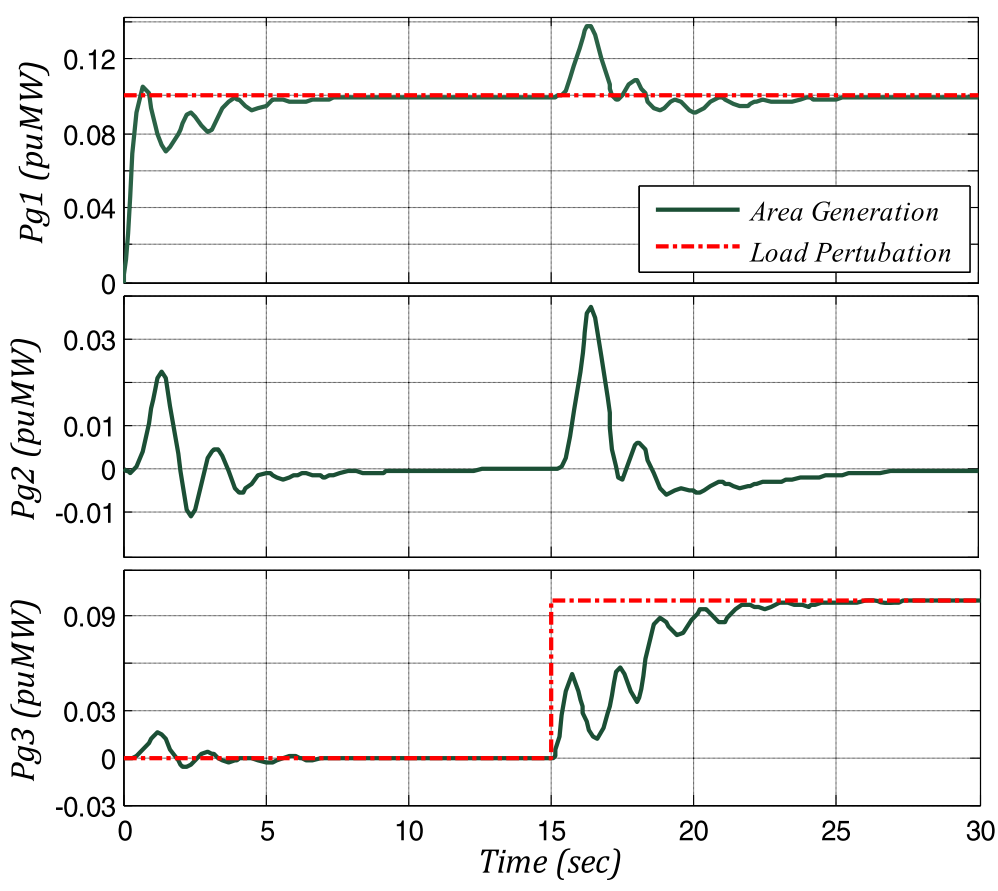

Fig. 7 Change in power generation 
with a reduced undershoot and settling time of $0.042 \mathrm{~Hz}$ and $5.33 \mathrm{~s}$ for the first load disturbance. This is a significant improvement when compared with the case of LFC loop alone (uncoordinated) which restores the frequency deviation with undershoot and settling time of $0.152 \mathrm{~Hz}$ and $7.58 \mathrm{~s}$ respectively.

The tie-line power deviations, as one of the states, are equally restored to their steady state within a short time as shown in Fig. 5. As in the case of frequency, coordinating the control loops has remarkably shorten the tieline power settling time and reduced the peak over/ undershoot for both load changes. For instance in $\mathrm{CA}_{3}$, the coordinated dis-MPC scheme restored the tie-line power deviations with undershoot and settling time of 0.095 puMW and 6.04 s for the second load disturbance representing $67.5 \%$ and $22.4 \%$ improvements over the uncoordinated case respectively.

Table 1 summarizes the improvement of the proposed control scheme over the LFC loops alone for the first load perturbation at $\mathrm{CA}_{1}$. The dynamic responses of the ACEs of the three CAs are shown Fig. 6.

It can be noted that following the application of 0.1 puMW step load perturbation at $\mathrm{CA}_{1}$, power flows from $\mathrm{CA}_{2}$, and $\mathrm{CA}_{3}$ to $\mathrm{CA}_{1}$ to cater for the load changes. However, after reaching a steady state, the increased power demand is supplied from $\mathrm{CA}_{1}$ only as shown in Fig. 7.

Similarly, for the second 0.1 puMW step load perturbation at $\mathrm{CA}_{3}$ (after $15 \mathrm{~s}$ ), the increased power demand is supplied from $\mathrm{CA}_{3}$ only at steady state.

\subsection{Random load perturbation}

$\mathrm{CA}_{1}$ and $\mathrm{CA}_{3}$ are simultaneously subjected to a random load perturbation (RLP) shown in Fig. 8, at the beginning of the simulation time.

The load change depicted in Fig. 8 is generated using the same technique as in [17]. It represents a more realistic and intermittently fluctuating load disturbance than MASP.

Load disturbance rejection performance of the proposed dis-MPC scheme is evaluated. As it can be observed from Figs. 9 and 10, both control schemes have maintained the frequency and tie-line power deviations within limits. However, the proposed coordinated control scheme has outperformed the uncoordinated with less fluctuation in the responses.

Furthermore, the responses of the area frequencies are compared with those obtained using quasi-oppositional whale optimization algorithm (QOWOA) proposed in [21]. This is to ascertain the superiority of the developed LFC scheme over-optimized classical controllers. To establish the basis for the comparison, corresponding minimum, maximum, mean, variance, and standard deviation (STDev) values of ITAE are computed and compared with those in [21] as summarized in Table 2.

\section{Discussion}

It is inferred from the system responses that the load disturbance rejection performance of the proposed disMPC scheme is demonstrated with and without the coordination between the AVR and LFC control loops. It is observed that, in either case, the frequency control schemes were able to maintain the system to their allowable ranges with negligible zero steady-state error. Nevertheless, coordinating the two control loops has generally shorten the settling times of all the measurable states and at the same time reduced the peak maximum deviations. The performance of the proposed scheme has reached the extent of rejecting the adverse effect of not only step load disturbance in multiple CAs but also random load disturbances. However, due to the intermittent nature of the random load disturbances, fast convergence of the dis-MPC cannot be guaranteed as in the case of step load disturbance.

The lower values of STDev and variance of the ITAE signify the robustness of the developed algorithm. It can therefore be deduced that the developed LFC scheme is more robust compared to the QOWOA-based with $23.21 \%$ improvement in the ITAE variance and $20.83 \%$ in the ITAE STDev of the ACE.

The significant improvement in the frequency control achieved by introducing the voltage control via AVR loop is a result of having more control input to the system. However, this increased number of the control

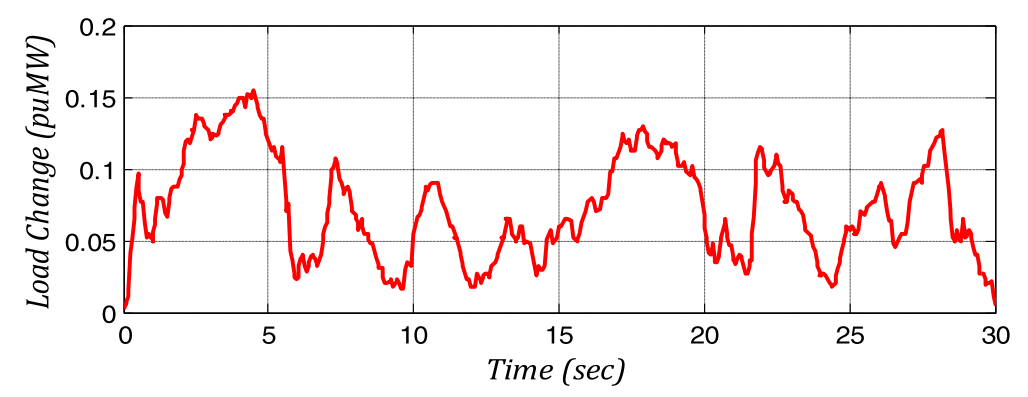

Fig. 8 Random load perturbation profile 


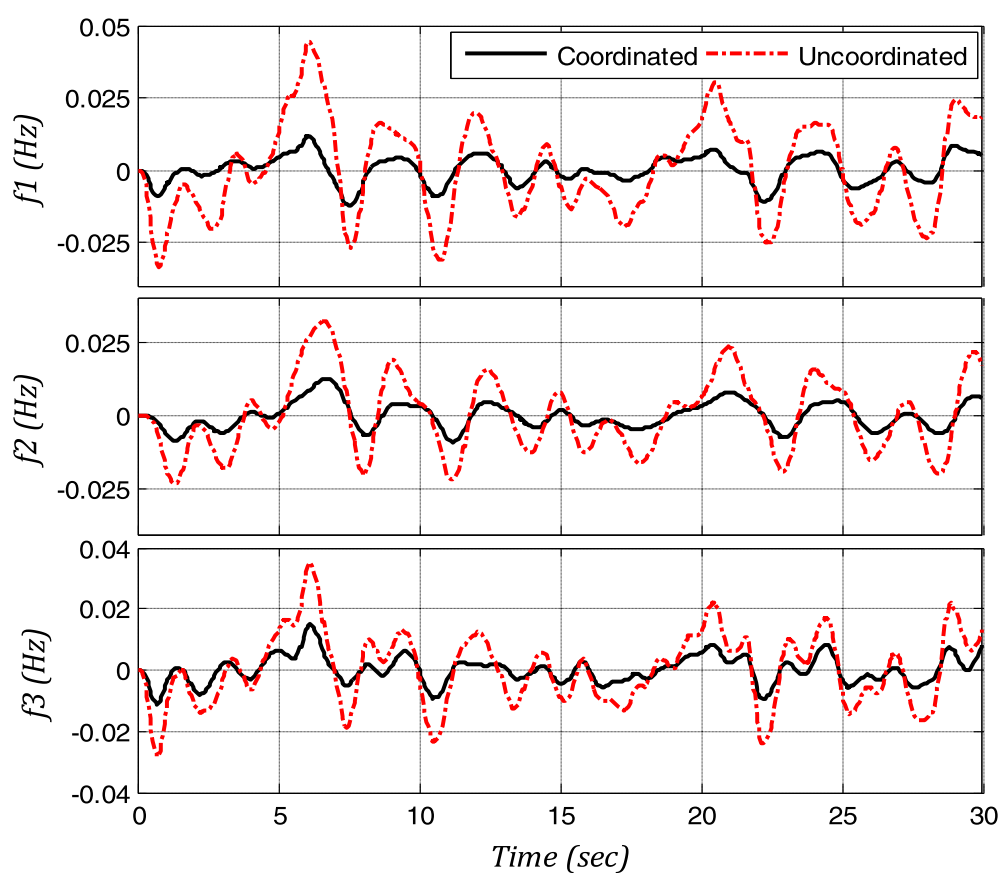

Fig. 9 Frequency deviations of the 3-CAs due to RLP

inputs has placed more computational burden on the MPC. It should be noted that the practical implementation of dis-MPC is still challenging due to the excessive computational burden caused by doubling the size of the control vector and long prediction horizon.

\section{Conclusion}

In this paper, LFC in IPS is carried out by synchronizing the LFC loop with that of AVR. Even though the AVR loop is substantially faster than the LFC loop, however, by the introduction of slow optimal control action on
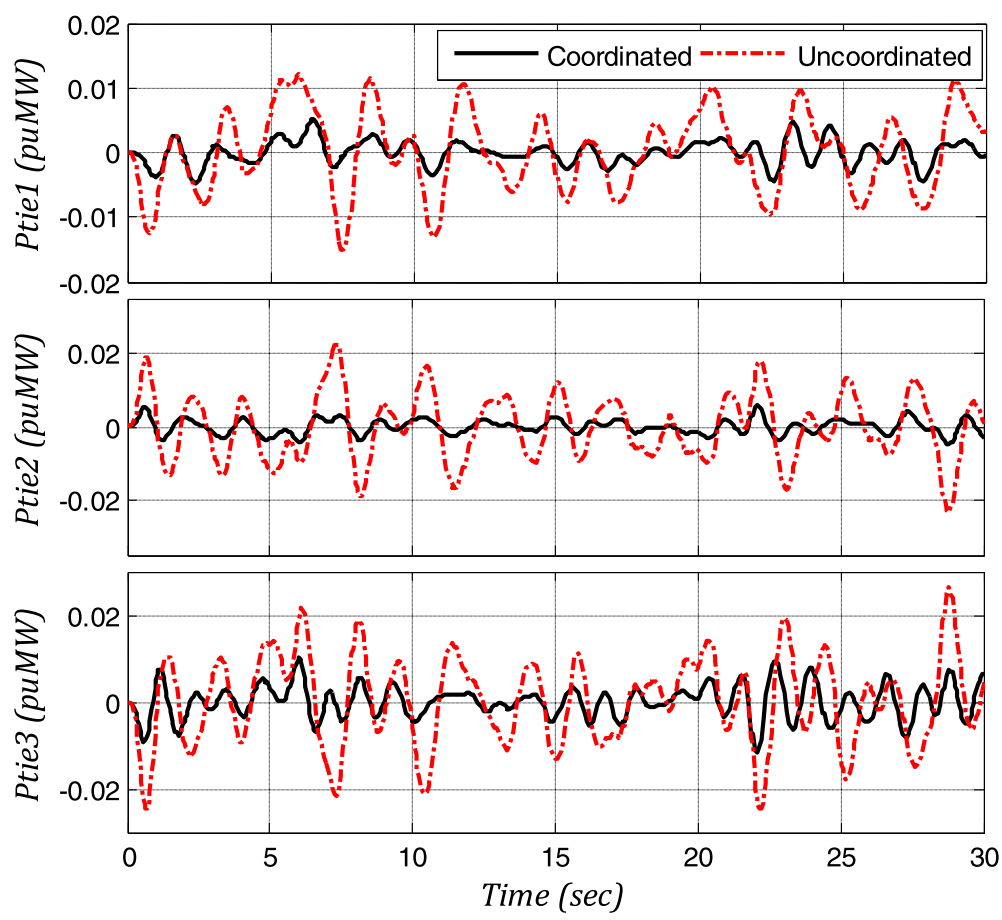

Fig. 10 Tie-line power deviations of the 3-CAs due to RLP 
Table 2 Performance measures for 20 runs of ITAE values

\begin{tabular}{llll}
\hline & QOWOA-based & dis-MPC based & Improvement (\%) \\
\hline Minimum & 0.0759 & 0.0589 & 22.34 \\
Maximum & 0.0898 & 0.0728 & 18.95 \\
Mean & 0.08055 & 0.06118 & 24.05 \\
Variance & $1.64 \times 10^{-5}$ & $1.26 \times 10^{-5}$ & 23.21 \\
STDev & 0.003214 & 0.003247 & 20.83 \\
\hline
\end{tabular}

the AVR, a positive damping effect has been achieved on the LFC loop thereby improving the frequency control. MPC is used as the supplementary controller. The work presents some of the advantages and applications of MPC to LFC, such as flexibility and coordination between multiple inputs. The dynamics of the IPS subjected to MASP and RLP are studied. The effectiveness of the proposed scheme is verified through time-based simulations. Based on the comparative analysis on the system responses, it is established that synchronizing the two control loops has improved the frequency control. It is observed that frequency and tie-line power maximum deviations have reduced by $66.4 \%$ and $59.09 \%$ in $\mathrm{CA}_{1}$, $32.75 \%$ and $58.53 \%$ in $\mathrm{CA}_{2}, 44.68 \%$ and $67.39 \%$ in $\mathrm{CA}_{3}$ respectively. While the respective settling times are reduced by $18.32 \%$ and $22.75 \%$ in $\mathrm{CA}_{1}, 18.32 \%$ and $22.75 \%$ in $\mathrm{CA}_{2}, 20.23 \%$ and $18.35 \%$ in $\mathrm{CA}_{3}$ respectively. While the robustness of the developed scheme is verified by comparing the STDev and variance of the response ITAE with those obtained using QOWOA-based LFC.

\begin{abstract}
Abbreviations
ACE: Area control error; AVR: Automatic voltage regulation; BFOA: Bacterial foraging optimization algorithm; CA: Control areas; dis-MPC: Distributed MPC; GA: Genetic algorithm; GRC: Generation rate constraints; IPS: Interconnected power system; ITAE: Integral time absolute error; LFC: Load frequency control; MASP: Multi-area step perturbation; MPC: Model predictive controller; OF: Objective function; PID: Proportional-integral-derivative; QOWOA: Quasioppositional whale optimization algorithm; RLP: Random load perturbation
\end{abstract}

\section{Acknowledgements}

Not applicable

\section{Authors' contributions}

All the authors contributed immensely to the success of this study. For instance, ABK designed the mathematical model of the three area interconnected power system (IPS), he further came up with the state-space model of the system. MA, in addition to some other minor contributions, model the coordination of the load frequency control (LFC) loop with that of the automatic voltage regulated (AVR). The major contribution credited to YAA is the design of the dense dis-MPC controller for the LFC application. He equally estimated the states of the CAs using Kalman filter. While YA contributed by formulating the constraints imposed on the system-like manipulated inputs, outputs, or state variables. He also contributed in analyzing the results, drawing inferences, proofreading the manuscript among others. All authors read and approved the final manuscript.

\section{Funding}

None.

Availability of data and materials Not applicable.
Ethics approval and consent to participate

Not applicable.

\section{Consent for publication}

Not applicable.

\section{Competing interests}

The authors declare that no competing interests are associated with the findings and the article itself.

\section{Author details}

${ }^{1}$ Department of Electrical Engineering, Ahmadu Bello University, Zaria, Nigeria. ${ }^{2}$ Engineering Program, Houston Community College, Texas, USA. ${ }^{3}$ Department of Computer Engineering, Ahmadu Bello University, Zaria, Nigeria.

Received: 9 October 2019 Accepted: 11 August 2020

Published online: 03 November 2020

\section{References}

1. Zheng Y, Zhou J, Xu Y, Zhang Y, Qian Z (2017) A distributed model predictive control based load frequency control scheme for multi-area interconnected power system using discrete-time Laguerre function. ISA Trans. 4:1-14

2. Shree SB, Kamaraj N (2016) Hybrid neuro fuzzy approach for automatic generation control in restructured power system. Int J Electr Power Energy Syst 74:274-285

3. Elgard Ol (1976) Electric energy system theory: an introduction. Tata McGraw-Hill Publishing Co., NY

4. Saadat H (1999) Power system control, in POWER SYSTEM ANALYSIS. MC Graw Hill, New York, pp 527-576

5. Ejegi E., Rossiter J. \& Trodden P., "Model predictive load frequency control of a two-area deregulated power system," European Control Conference (ECC), Linz, Austria, pp. 1044 - 1049, 2015.

6. Wood AJ, Wollenberg BF, Sheblé GB (2014) Power generation operation and control. John Wiley, Newyork

7. Hemmati R, Azizi N, Khah M, Catalao J (2018) Decentralized frequencyvoltage control and stability enhancement of standalone wind turbine-loadbattery. J Electr Power Energy System 102:1-10

8. Ma M, Chen H, Liu X, Allgöwer F (2014) Distributed model predictive load frequency control of multi-area interconnected power system. Electr. Power Energy Syst. 62:289-298

9. Bevrani H, Mitani Y, Tsuji K, Bevrani \& Bevrani H. (2005) Bilateral based robust load frequency control. J Energy Conversion Manag 46(7):1129-1146

10. Shayeghi H, Malik OP (2007) Robust decentralized neural networks based LFC in a deregulated power system. Power System Research 77(4):241-251

11. Tan W, Hao Y, Li D (2015) Load frequency control in deregulated environments via active disturbance rejection. Int J Electr Power Energy Syst 66:166-177

12. Saxena S, Hote $\mathrm{W}$ (2017) Stabilization of perturbed system via IMC: an application to load frequency control. Control Eng Pract 64:61-73

13. Kouba N. Y., Menaa M., Hasni M. \& Boudour M., "Load frequency control in multi-area power system based on fuzzy logic-PID controller," IEEE International Conference on Smart Energy Grid Engineering (SEGE), Oshawa, ON, Canada, 2015

14. Hou G., Qin L., Zheng X. and Zhang J., "Design of PSO-based fuzzy gain scheduling PI controller for four-area interconnected AGC system after deregulation," International Conference on Advanced Mechatronic Systems, Zhengzhou, China, 2011

15. Parmar S. M. \& Hiskens K., "LFC of an interconnected power system with multi-source power generation in deregulated power environment" Int'| Journal of Electr. Power \& Energy System, vol. 57, pp. pp. 277-286, 2014.

16. Ersdal A., Imsland L., \& Uhlen L., "Distributed model predictive load frequency control of a deregulated power system" Belfast, UK., 2016.

17. Hermans R. M., Jokić A., Lazar M., Alessio A, Paul P.J, lan A, Hiskens I. A. \& Alberto Bemporad A., "Assessment of non-centralised model predictive control techniques for electrical power networks," International Journal of Control, vol. 85, no. Taylor and Francis, p. 1162-1177, 2013.

18. Shiroei M, Ranjbar A (2014) Supervisory predictive control of power system load frequency control. Int J Electrical Power Energy System 61:70-80 
19. Abd-Elazim S. M. and Ali E. S., "Optimal PID tuning for load frequency control using bacteria foraging optimization algorithm," Cairo, 2010.

20. Saikia S (2015) Automatic generation control of a multi-area thermal power system using grey wolf optimizer algorithm based classical controllers. Electr. Power Energy Syst. 73(2):853-862

21. Simhadri KS, Mohanty B (2019) Performance analysis of dual-mode PI controller using quasi-oppositional whale optimization algorithm for load frequency control. Int Transac Electr Energy System:1-23

22. Guha D, Roy PK, Banerjee S (2016) Quasi-oppositional differential search algorithm applied to load frequency control. Int J Eng Sci Technol 19(4): $1635-1654$

23. Ellithy K, Said S, Kahlout O (2014) Design of power system stabilizers based on $\mu$-controller for power system stability enhancement. Electr. Power Energy Syst. 63:933-939

24. Su H, Liu T (2018) WAMS-based coordinated automatic voltage regulation incorporating voltage stability constraints using sequential linear programming approximation algorithm. Electr. Pow. Syst. Res. 163:482-490

25. Stil VJ, Mehmedovic M (2018) Interconnection and damping assignment automatic voltage regulator for synchronous generators. Electr. Power Energy Syst. 101:204-212

26. Ahmadi S, Shokoohi S, Bevrani H (2015) A fuzzy logic-based droop control for simultaneous voltage and frequency regulation in an AC microgrid. Electr. Power Energy Syst. 64:148-155

27. Hirase Y, Abe K, Ise T (2018) A novel control approach for virtual synchronous generators to suppress frequency and voltage fluctuations in mirogrids. Appl. Energy 210:699-710

28. Ejegi EE, Rossiter JA, Trodden P (2016) Distributed model predictive load frequency control of a deregulated power system. Belfast, UK

29. Elsisi M, Soliman M, Aboelela MAS, Mansour W (2016) Bat inspired algorithm based optimal design of model predictive load frequency control. Electr. Power Energy Syst.:426-433

\section{Publisher's Note}

Springer Nature remains neutral with regard to jurisdictional claims in published maps and institutional affiliations.

\section{Submit your manuscript to a SpringerOpen ${ }^{\circ}$ journal and benefit from:}

- Convenient online submission

- Rigorous peer review

- Open access: articles freely available online

High visibility within the field

- Retaining the copyright to your article

Submit your next manuscript at $\boldsymbol{\nabla}$ springeropen.com 